

Boenke, Patrick (2003/05): Zur amerikanischen Rezeption der Schichtenlehre Heinrich Schenkers. ZGMTH 1-2/2/2-3, 181-188. https://doi.org/10.31751/525

(C) 2003/05 Patrick Boenke

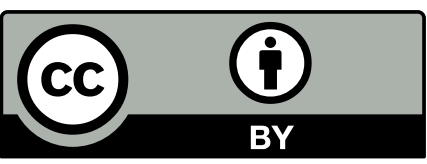

Dieser Text erscheint im Open Access und ist lizenziert unter einer Creative Commons Namensnennung 4.0 International Lizenz.

This is an open access article licensed under a

Creative Commons Attribution 4.0 International License.

veröffentlicht / first published: 01/04/2005

zuletzt geändert / last updated: 15/05/2013 


\title{
Zur amerikanischen Rezeption der Schichtenlehre Heinrich Schenkers
}

\author{
Patrick Boenke
}

Die Vertreibungspolitik der Nazis hatte zur Folge, daß der überwiegende Teil der europäischen Schenkeristen nach Nordamerika flüchtete. Der an der renommierten `Mannes Schook in New York lehrende Hans Weisse und sein Nachfolger Felix Salzer beeinflußten die Grundrichtung der frühen Schenker-Rezeption in Amerika maßgeblich. Sie unterzogen die Schichtentheorie Schenkers einer weitreichenden Revision, insbesondere um sie den akademischen Erfordernissen in Amerika anzupassen. Orthodoxe Schenkerianer wie Oswald Jonas und Ernst Oster dagegen verbreiteten die Schichtenlehre vornehmlich auf dem Wege des Privatunterrichts. Sowohl die Reformer als auch die orthodoxen Kräfte lösten eine nachhaltige Rezeption der Theorie Schenkers in Nordamerika aus. In der Folge etablierte sich die Schichtenlehre als die vorherrschende Theorie für die Analyse tonaler Musik. Mit dem Vorrücken amerikanischer Verfechter der Schichtenlehre - unter ihnen Allen Forte und Carl Schachter - auf einflußreiche akademische Lehrpositionen entwickelte sich die Schenker-Analyse zu einem eigenständigen Forschungsgebiet der amerikanischen smusic theorys.

Die besondere Situation, daß die Schichtenlehre Heinrich Schenkers in Nordamerika eine herausragende Stellung einnimmt, hingegen im europäischen Raum nur vereinzelt aufgenommen wurde, ist zu einem wesentlichen Teil der Vertreibungspolitik der Nazis in den 1930er Jahren zuzuschreiben', die die meisten Mitglieder des europäischen Schenker-Kreises - unter ihnen Moritz Violin, Felix Salzer, Oswald Jonas und Ernst Oster - in das Exil zwang. Allein Hans Weisse, der bereits 1931 an der >David Mannes Music Schook in New York zu unterrichten begann (Grünzweig 1993), war ohne äußeren Druck emigriert. Seine Einstellung zur Lehre Schenkers prägte die Grundhaltung der frühen Schenker-Rezeption in den USA maßgeblich. Bereits in den 1930er Jahren polarisierte ein grundsätzlicher Richtungsstreit den europäischen Schenkerismus. Vertraten Moritz Violin und Oswald Jonas eine orthodoxe Position, die Schenkers Lehre allenfalls von Polemik und politischem Ballast zu befreien suchte, so strebten Hans Weisse und Felix Salzer eine Neuformulierung an. Schenkers posthum veröffentlichtes Hauptwerk Der Freie Satz (1935) erschien ihnen aus pädagogischen und systematischen Gesichtspunkten ungeeignet, einen größeren Interessentenkreis für die Schichtenlehre zu gewinnen. Nach ersten Lehrerfahrungen in New York empfahl Weisse denn auch dem auf der Flucht befindlichen Jonas in einem Brief vom 17. 7. 1938: »Lassen Sie die absolut konzes-

1 Vgl. Schwab-Felisch 2005, $373 f$. 
sionslose und zu polemische Seite der Schenkerlehre fallen und bringen Sie Ihr Wissen ohne den Fehdehandschuh herüber.«² Weisse spürte, daß Schenkers Dogmatismus und reaktionäre Polemik unter den akademischen Bedingungen, die er in Amerika vorfand, nicht auf fruchtbaren Boden stoßen konnten. Schenkers Theorie in der Konsequenz zu ssäkularisieren und in systematischer Gliederung zu lehren hieß jedoch, sie aus dem elitären Zirkel der Eingeweihten herauszuheben und einem größeren Kreis zu öffnen. Dies mußte orthodoxe Schenkerianer wie Jonas oder Oster zum Widerstand aufrufen ${ }^{3}$, denn es war zu befürchten, daß Schenkers Kunstlehre unter den Reformbestrebungen als systematisch lehrbare Methode mißdeutet wurde. Da sowohl Jonas als auch Oster über Jahre hinweg eine akademische Karriere verwehrt blieb, konnten sie nur auf dem Wege des Privatunterrichts versuchen, die Schichtenlehre in unangetasteter Form zu bewahren. ${ }^{4}$

Nach Weisses Tod im Jahre 1940 wurde Felix Salzer als sein Nachfolger bestellt. Er teilte die Bedenken seines Vorgängers, daß die Schichtenlehre nur in modifizierter Form, nicht aber in der Darstellung von Der Freie Satz den ihr gebührenden Platz in der akademischen Ausbildung finden könnte. Schenkers schwer zugänglicher Sprachstil, die unvollständige, bisweilen sogar widersprüchliche Entwicklung seiner Ideen und das Fehlen einer pädagogischen Systematik machten in Salzers Augen eine tiefgehende Revision der Schichtenlehre unumgänglich. ${ }^{5}$ Auch der wiederholt erhobenen Forderung, die Schriften Schenkers in englischen Übersetzungen bereitzustellen, begegnete Salzer mit begründetem Zweifel. Wie vor ihm schon Weisse befürchtete er, daß Schenkers apodiktischer Tonfall im Umfeld der aufstrebenden amerikanischen Musiktheorie, die mit positivistischen Idealen gerade zur Verwissenschaftlichung drängte, mehr zu seiner Ablehnung denn zu seiner Annahme und Verbreitung beitrüge. ${ }^{6}$ In seiner Lehrtätigkeit an der »Mannes School« dagegen war es Salzers Verdienst, die Theorie Schenkers im Curriculum fest zu verankern. ${ }^{7}$ Sein 1952 veröffentlichtes Lehrbuch Structural Hearing. Tonal Coherence in Music (1952)- Resultat seiner langjährigen Unterrichtserfahrung - avancierte zu einer weithin anerkannten Einführung in die Theorie Schenkers. Über Jahre hinweg blieb es eine wichtige Quelle, die eine breitere Auseinandersetzung mit der

2 Zit. nach Kurth 1985, 113.

3 Die Korrespondenz insbesondere mit Salzer belegt heftige Auseinandersetzungen. Diese führten letztlich zum endgültigen Zerwürfnis.

4 Insbesondere Oster prägte die ersten Generationen amerikanischer Schenkerianer nachhaltig. Zeitweilige Lehrverpflichtungen führten ihn auch an die sMannes Schook. Oswald Jonas unterrichtete bis in die Mitte der 1960er Jahre an der >Roosevelt University، in Chicago und wechselte anschließend an die ,University of Californias, Riverside.

5 Bereits 1935 fragte Weisse: »(S)oll nach Schenker gelehrt werden - stellt die jetzige Gestalt des freien Satzes alles in allem sich einem solchen Zwecke zur Verfügung? Oder bedarf es noch der Erklärung und Erläuterung, und in manchen Fällen, besonders was die präzise Abgrenzung der Begriffe anlangt, sogar auch der Richtigstellung?« (Zit. nach Kurth 1985, 101).

6 Erst verhältnismäßig spät - seit den 1970er Jahren - wurden größere Teile der Schriften Schenkers in englischer Übertragung zugänglich gemacht.

7 Mit einer Vielzahl herausragender Lehrer und Absolventen - unter ihnen Allen Forte und Carl Schachter - blieb sMannes` über Jahre hinweg ein Zentrum des nordamerikanischen Schenkerismus. 
Schichtenlehre ermöglichte und beförderte, zugleich aber die Rezeption der originalen Schriften erschwerte, da Salzers Text nicht explizit erkennen ließ, an welchen Punkten er von Schenkers Darstellung abwich und seine Ideen modifizierte, erweiterte oder gar korrigierte. Erst im Jahre 1979 erschien dann die lang ersehnte englische Übersetzung Free Composition, die Oster über Jahre hinweg vorbereitet hatte. Doch auch sie ermöglichte nur bedingt einen authentischen Zugang zu Schenkers Schichtenlehre, da sich Oster - wie vor ihm schon Jonas in der zweiten deutschsprachigen Auflage von Der Freie Satz ${ }^{8}$ - für eine weitgehende Zensur entschied, die den originalen Text von allen politisch verfänglichen Passagen befreite. ${ }^{9}$

Dem Komponisten Milton Babbitt sowie dem Theoretiker Allen Forte ${ }^{10}$ war es zu verdanken, daß die Theorie Schenkers Einzug in die universitären Curricula der `Ivy League hielt. Babbitt trat entschieden für die Fortsetzung des von Weisse und Salzer eingeschlagenen Weges der Versachlichung ein. In seiner Rezension von Salzers Structural Hearing pointierte er seine Haltung mit der Forderung, Schenkers politische und gesellschaftliche Äußerungen und sein verzerrtes Geschichtsbild vollends vom eigentlichen Korpus der Theorie zu lösen. ${ }^{11}$ Andere - beispielsweise Michael Mann (1949) - sahen sich dagegen außerstande, von den ideologischen Zügen der Theorie abzusehen. Bis in die gegenwärtige Zeit hinein erschwert Schenkers kultur- und gesellschaftspolitische Weltsicht ${ }^{12}$ die unbefangene Aufnahme seiner Theorie..$^{13}$

Beschränkte sich Babbitt auf einige wenige schriftliche Äußerungen zur Theorie Schenkers, so entfaltete Allen Forte eine rege Publikationstätigkeit. ${ }^{14}$ Sein früher Aufsatz "Schenker's Conception of Musical Structure» (1959) geht in den einleitenden Bemerkungen auf viele Vorbehalte und Mißverständnisse ein, welche die Schenker-Rezeption der 1950er Jahre grundsätzlich charakterisierten. Den Kritikern der Schichtenlehre versuchte Forte in der Folge mit größtmöglicher Neutralität und Sachlichkeit zu begegnen. Als richtungsweisend erwies sich eine den Aufsatz abschließende Zusammenstellung fünf ungelöster Probleme der Musiktheorie. Das letzte unter ihnen - »understanding the structure of problematic modern works $\ll^{15}$ - erscheint insofern bemerkenswert, als Forte dort den Versuch unternimmt, die Schichtenlehre außerhalb der von Schenker selbst gesetzten historischen Grenzen anzuwenden. Die Überlegung, bestimmte ihrer

8 Die von Oswald Jonas herausgegebene und bearbeitete zweite Auflage erschien 1956.

9 Zur Problematik der Eingriffe vgl. Rothstein 1990a, 195f.

10 Mit Allen Forte gewann der amerikanische Schenkerismus einen seiner einflußreichsten Lehrer. Forte wurde zu Beginn der 1950er Jahre durch Alvin Baumann, einen Schüler von Weisse, in die Theorie Schenkers eingeführt. Unter Fortes Schülern befinden sich mit David Beach, David Carson Berry, William Caplin, Janet Schmalfeldt und William Rothstein eine Reihe prominenter Theoretiker (ich danke Sigrun Heinzelmann für diese Informationen, die sie in persönlichen Gesprächen mit amerikanischen Schenkeristen zusammengetragen hat).

11 Babbitt 1952, 264.

12 Siehe hierzu das erste Kapitel in Eybl 1995.

13 Vgl. Rothstein 1990a.

14 Mit Steven E. Gilbert verfaßte er 1982 ein Lehrbuch zur Schenker-Analyse. Insbesondere wandte sich Forte auch Fragen der Analyse atonaler Musik des 20. Jahrhunderts zu.

15 Ebd., 25ff. 
Teilstücke auch für Musik vor 1700 bzw. nach 1900 in Anspruch zu nehmen, war indes nicht neu. ${ }^{16}$ In Fortes spezifischem Zugang jedoch geriet die Schenker-Analyse zunehmend unter den Einfluß analytischer Methodiken der atonalen Musik des 20. Jahrhunderts. Seine späteren Analysen demonstrieren einen shybriden Ansatz $^{17}$, in welchem er Schenkers hierarchisches Schichtenmodell mit seiner in den 1970er Jahren entwickelten spitch-class-set $\_-$Theorie $^{18}$ verband.

Überlegungen, den Geltungsbereich der Schenker-Theorie durch Modifikationen zu erweitern, blieben ein bestimmendes, wenngleich kontrovers diskutiertes Motiv in der Auseinandersetzung mit Schenker. In dem Maße, wie Teilstücke seiner Theorie - beispielsweise das Konzept hierarchisch bezogener Schichten oder aber die Vorstellung der `Auskomponierung` von Klängen - auf Werke außerhalb der von Schenker betrachteten Zeitspanne angewendet wurden, mußten andere zentrale Aspekte, insbesondere die Theorie des ıUrsatzes`, abgeschwächt oder gar ganz aufgegeben werden. Je weiter das Zeitfenster geöffnet wurde, um so stärker konnten einzelne Ideen Schenkers allgemeine und epochenübergreifende Gültigkeit beweisen. Als Kehrseite dessen wurde jedoch die Theorie in ihren Fundamenten ausgehöhlt.

Neben Versuchen der Erweiterung orientierte sich eine große Anzahl insbesondere werkanalytischer Beiträge weiterhin an dem von Schenker abgesteckten historischen Rahmen. Die besondere Stellung analytischer Werkstudien in der amerikanischen Musiktheorie mag - aus europäischer Sicht - zunächst befremden. Verständlich wird sie, führt man sich die gänzlich anders gelagerten Rahmenbedingungen vor Augen, unter denen sich in Amerika eine smusic theory akademischer Prägung entwickelte. Blieb der Theorie, und mit ihr der Analyse, im Fächerkanon der europäischen Musikwissenschaft ein wissenschaftlicher Status versagt, so konnte sie sich umgekehrt in Amerika unter dem vorherrschenden Wissenschaftsideal der 1950er und 1960er Jahre als eigenständige Disziplin behaupten. Schenkers werkanalytische Tradition, die sich neben seinen Monographien und Erläuterungsausgaben in den zahllosen Analysen des Periodikums Der Tonwille und der drei Jahrbücher Das Meisterwerk in der Musik niederschlug, konnte unter diesen Umständen ungehindert ihre Fortsetzung finden.

Mit zunehmender Akzeptanz und Verbreitung differenzierten sich die Arbeitsfelder der amerikanischen Schenker-Forschung. ${ }^{19}$ Neben Fragen der Methodologie und päd-

16 Siehe neben Salzer 1952 auch Katz 1945. - Bezeichnenderweise wurden ähnliche Gedanken auch in den nur vereinzelten Ansätzen der deutschsprachigen Rezeption aufgeworfen: Hellmut Federhofer - bedeutender Vertreter der Theorien Schenkers im deutschsprachigen Raum - verwies im besonderen darauf, daß die »Reduktionstechnik« zur Aufdeckung der musikalischen »Verlaufsgestalt« nicht allein in den von Schenker gezogenen historischen Grenzen anwendbar sei - siehe »Reduktionstechnik und Gestaltanalyse«, in: Federhofer 1950, 1-5.

17 Vgl. etwa Forte 1987. Die Tatsache, daß die Schichtenlehre Einzug in die Lehrpläne der >Undergraduate-Ausbildung hielt, gab nachhaltige Impulse für einen pädagogischen Diskurs.

18 Forte 1973. - Vgl. auch Scheideler 2005.

19 Die schrittweise aktualisierten Bibliographien von David Beach dokumentieren umfassend diesen Prozeß (Beach 1969, 1979, 1985, 1989). Vgl. des weiteren Berry 2004. 
agogischen Vermittlung ${ }^{20}$ wandte man sich nun auch den spezielleren Aspekten der Theorie und ihrer geistesgeschichtlichen Entwicklung zu: Die Evolution des Ursatzmodells in den Schriften Schenker (Pastille 1990a), ihre ideengeschichtliche Verwurzelung in der Goetheschen Morphologie (Pastille 1990b) und die wiederholt von Schenker bemühte Metaphorik des `Organischen ${ }^{21}$ bildeten den Ausgangspunkt umfassender Forschungen. Weiterhin rückten all jene Bereiche in das Blickfeld, die Schenker in seinem theoretischen OEuvre nur skizzenhaft hinterließ. ${ }^{22}$

So erwiesen sich Schenkers knappe Ausführungen zur Rhythmik und Metrik in Der Freie Satz als tragfähiges Fundament, das Carl Schachter in seiner Trilogie »Rhythm and Linear Analysis ${ }^{23}$ und William Rothstein in seiner Dissertation »Rhythm and the Theory of Structural Levels $\aleph^{24}$ zu einer konsistenten Theorie ausbauten. Bereits in den 1950er Jahren hatte Allen Forte die Entwicklung einer rhythmischen Theorie der tonalen Musik als eine der dringlichsten Fragestellungen der Musiktheorie ausgerufen. ${ }^{25}$

Weiterhin versuchte man, Schenkers versprengte Äußerungen zur Motivik zu einem schlüssigen Bild zusammenzufügen. Charles Burkharts richtungsweisende Studie "Schenker's >Motivic Parallelisms $«^{26}$ legte den Grundstein für die Ausarbeitung einer Motivtheorie in der Perspektive Schenkers. Bereits der sparsame Gebrauch des Terminus >Motiv in Schenkers eigenen Analysen ließ auf die Bedenken schließen, die er den landläufigen Motivauffassungen seiner Zeit entgegenbrachte. Sein Interesse galt weniger dem Motivgeflecht der musikalischen Oberfläche als den ıverborgenen Wiederholungen`, die sich insbesondere auch zwischen hierarchisch differenten Schichten einstellen konnten. ${ }^{27}$ Erste Prolongationen des ১Ursatzes`, auf die Allen Cadwallader (1988) seinen Blick richtete, wirken somit nicht allein in die schichtweise organisierte tonale Struktur des einzelnen Werkes hinein, sondern können zugleich als Motive erster Ordnung maßgeblich die motivischen Entfaltungen hierarchisch niedrigerer Schichten beeinflussen. Die Einsicht, daß Motivik in der tonalen Musik in vergleichbarer Weise hierarchisch strukturiert sein kann wie Tonalität, lenkte das analytische Interesse auf eine weitere Tiefendimension des tonalen Satzes und deckte ein von den flachen Bezügen der musikalischen Oberfläche zu unterscheidendes Beziehungsnetz auf.

Ein drittes Forschungsfeld betraf Schenkers Ausarbeitungen zur Form. ${ }^{28}$ Wurde in traditionellen Zugängen Form primär als Segmentierung der musikalischen Oberfläche

20 Die Tatsache, daß die Schichtenlehre Einzug in die Lehrpläne der `Undergraduate-Ausbildung hielt, gab nachhaltige Impulse für einen pädagogischen Diskurs.

21 Solie 1980 und Kassler 1983.

22 Vgl. Schwab-Felisch 2005, 354-66.

23 Schachter 1976, 1980, 1987.

24 Rothstein 1981, vgl. auch Rothstein 1990b. Als Weiterführung dieser Studien vgl. Rothstein 1989.

25 Vgl. Forte 1959, $20 f$.

26 Burkhart 1978. Vgl. auch Rothgeb 1983.

27 Motive höherer Ordnung sind einer tieferen Schicht zugehörig und entziehen sich allein schon dadurch weitgehend einem tradierten Motivbegriff, da sie zumeist auf einfachsten Tonkonstellationen beruhen und rhythmisch unkonturiert bleiben.

28 Siehe das letzte Kapitel in Schenker 1935. 
verstanden, so entwickelte Schenker - vom musikalischen `Hintergrund geleitet - ein abweichendes Verständnis, in dem Form als Resultat der über die Schichten hinweg zunehmend komplexeren Ausdifferenzierung des `Ursatzes` begriffen wurde. Der unterschiedliche Blickwinkel beider Perspektiven offenbarte sich mitunter im oftmals spannungsvollen Verhältnis von äußerer und innerer Form, d. h. von oberflächlicher Tektonik und ihr unterliegender tonaler Struktur im Sinne Schenkers. ${ }^{29}$ Einen wichtigen Schritt hin zur Entwicklung einer Formtheorie in den Bahnen Schenkers unternahm Allen Cadwallader (1990), indem er die strukturkonstituierenden Merkmale einer einzelnen Schicht von Merkmalen des >Designs` der ihr nachfolgenden unterschied. Mag beispielsweise eine Nebennote in einer tieferen Schicht zunächst als individuelle Ausgestaltung eines Gerüsttones und damit als Merkmal des `Designs` erscheinen, so kann sie sich in einer späteren Schicht zu einer formkonstituierenden Komponente wandeln, indem sie sich im weiteren Verlauf der Auskomponierung etwa zu einem tonartlich kontrastierenden Teil der äußeren Gesamtform auswächst. In dieser Sichtweise konstituiert letztlich jede einzelne Schicht ihre eigene Form.

Angesichts der seit den 1980er Jahren immens angewachsenen Zahl an Publikationen, die sich der Theorie Schenkers widmen, können die vorhergehenden Anmerkungen nur als Schlaglichter auf einzelne Aspekte und Themenfelder angesehen werden. Der Überblick wird dadurch zusätzlich erschwert, daß Ideen Schenkers im Zuge des für die jüngere Rezeption charakteristischen Öffnungsprozesses verstärkt auch in interdisziplinär gelagerte Diskussionen eingebracht wurden. In der Folge wurden neue Arbeitsfelder erschlossen, die oft nur noch partiell jene Gebiete berührten, die Schenker in den Mittelpunkt seines Schaffens rückte: Analyse der Meisterwerke, Quellenstudium und Interpretation. So konnte die Schenker-Analyse unter dem Einfluß ,formalistischer Tendenzen $^{30}$ - als deren folgenreichste Entwicklung darf die `Generative Theory of Tonal Music des Komponisten Fred Lerdahl und des Linguisten Ray Jackendoff angesehen werden - bis hin zu automatisierten Verfahren der computergestützten Analyse vordringen. Ihr Gegengewicht fanden diese Entwicklungen in einer poststrukturalistisch geprägten Kritik, die ihr Wort nicht allein gegen Schenker erhob, sondern allgemein gegen eine primär an der Strukturanalyse interessierte Theoriebildung.

\section{Literatur}

Babbitt, Milton (1952), Rezension von Felix Salzer: Structural Hearing, Journal of the American Musicological Society 5, 260-65.

Beach, David (1969), „A Schenker Bibliography«, Journal of Music Theory 13, 2-37.

— (1979), »A Schenker Bibliography: 1969-1979«, Journal of Music Theory 23, 275-86.

(1985), »The Current State of Schenkerian Research«, Acta Musicologica 57, 275307.

29 Die Begriffe »outer« und »inner form« prägte Felix Salzer (1952, 223f.).

30 Vgl. Schwab-Felisch 2005, 372. 
_ (1989), „Schenkerian Theory«, Music Theory Spectrum 11, 3-14.

Berry, David Carson (2004), A Topical Guide to Schenkerian Literature, Hillsdale: Pendragon Press.

Burkhart, Charles (1978), „Schenker's >Motivic Parallelisms«", Journal of Music Theory 22, 145-75.

Cadwallader, Allen (1988), »Prolegomena to a General Description of Motivic Relationships in Tonal Music«, Intégral 2, 1-35.

_ (1990), »Form and Tonal Process. The Design of Different Structural Levels», in: Trends in Schenkerian Research, hg. von Allen Cadwallader, New York: Schirmer, $1-21$.

Eybl, Martin (1995), Ideologie und Methode. Zum ideengeschichtlichen Kontext von Schenkers Musiktheorie (Wiener Veröffentlichungen zur Musikwissenschaft 32), Tutzing: Hans Schneider.

Federhofer, Hellmut (1950), Beiträge zur musikalischen Gestaltanalyse, Graz: Akademische Druck- und Verlagsanstalt.

Forte, Allen (1959), „Schenker's Conception of Musical Structure«, Journal of Music Theory $3,1-30$.

— (1973), The Structure of Atonal Music, New Haven und London: Yale University Press.

(1987), „Liszt's Experimental Idiom and Music of the Early Twentieth Century«, 19th-Century Music 10, 209-28.

_ / Steven E. Gilbert (1982), Introduction to Schenkerian Analysis, New York und London: W. W. Norton.

Grünzweig, Werner (1993), »Vom `Schenkerismus` zum `Dahlhaus-Projekt««, Österreichische Musikzeitschrift 48, 161-70.

Kassler, Jamie Croy (1983), »Heinrich Schenker's Epistemology and Philosophy of Music. An Essay on the Relations between Evolutionary Theory and Music Theory", in: The Wider Domain of Evolutionary Thought, hg. von David Oldroyd und Ian Langham, Dordrecht: D. Reidel, 221-60.

Katz, Adele (1945), Challenge to Musical Tradition. A New Concept of Tonality, New York: Alfred A. Knopf.

Kurth, Ulrich (1985), »Die Auswirkungen der Lehre Heinrich Schenkers und seiner Schüler in den USA", Schlußbericht des Projektes der Deutschen Forschungsgemeinschaft Exilforschung. Wiener Schulen in den USA, Kiel (masch.).

Mann, Michael (1949), "Schenker's Contribution to Music Theory«, The Music Review 10, 3-26.

Pastille, William (1990a), »The Development of the Ursatz in Schenker's Published Works", in: Trends in Schenkerian Research, hg. von Allen Cadwallader, New York: Schirmer, 71-85. 
(1990b), »Music and Morphology. Goethe's Influence on Schenker's Thought», in: Schenker Studies, hg. von Hedi Siegel, Cambridge: Cambridge University Press, 29-44.

Rothgeb, John (1983), »Thematic Content. A Schenkerian View«, in: Aspects of Schenkerian Theory, hg. von David Beach, New Haven und London: Yale University Press, 39-60.

Rothstein, William (1981), »Rhythm and the Theory of Structural Levels«, diss., Yale University.

(1989), Phrase Rhythm in Tonal Music, New York: Schirmer.

_ (1990a), »The Americanization of Heinrich Schenker«, in: Schenker Studies, hg. von Hedi Siegel, Cambridge: Cambridge University Press, 193-203.

(1990b), »Rhythmic Displacement and Rhythmic Normalization«, in: Trends in Schenkerian Research, hg. von Allen Cadwallader, New York: Schirmer, 87-113.

Salzer, Felix (1952), Structural Hearing. Tonal Coherence in Music, New York: Charles Boni. Dt. als Strukturelles Hören. Der tonale Zusammenhang in der Musik, Wilhelmshaven: Heinrichshofen 1977.

Schachter, Carl (1976), „Rhythm and Linear Analysis. A Preliminary Study«, The Music Forum 4, 281-334.

_ (1980), »Rhythm and Linear Analysis. Durational Reduction«, The Music Forum 5, 197-232.

_ (1987), »Rhythm and Linear Analysis. Aspects of Meter«, The Music Forum 6, 159.

Scheideler, Ullrich (2005), »Analyse von Tonhöhenordnungen. Allen Fortes Pitch-ClassSet-System«, in: Handbuch der Systematischen Musikwissenschaft, Band 2: Musiktheorie, hg. von Helga de la Motte-Haber und Oliver Schwab-Felisch, Laaber: Laaber, 391-408.

Schenker, Heinrich (1935), Neue musikalische Theorien und Phantasien. 3. Der Freie Satz, Wien: Universal Edition.

— (1979), Free Composition, hg. und übersetzt von Ernst Oster, New York: Longman.

Solie, Ruth (1980), »The Living Work: Organicism and Musical Analysis«, 19th-Century Music 4, 147-56.

Schwab-Felisch, Oliver (2005), »Zur Schichtenlehre Heinrich Schenkers», in: Handbuch der Systematischen Musikwissenschaft, Band 2: Musiktheorie, hg. von Helga de la Motte-Haber und Oliver Schwab-Felisch, Laaber: Laaber, 337-76. 